\title{
Fruit Extract Mediated Green Synthesis of Metallic Nanoparticles: A New Avenue in Pomology Applications
}

\author{
Harsh Kumar ${ }^{1}\left(\mathbb{D}\right.$, Kanchan Bhardwaj ${ }^{2}$, Daljeet Singh Dhanjal ${ }^{3}\left(\mathbb{D}\right.$, Eugenie Nepovimova ${ }^{4}$, \\ Fatih Șen ${ }^{5}$, Hailemeleak Regassa ${ }^{6}$, Reena Singh ${ }^{3}$, Rachna Verma ${ }^{2}{ }^{(D}, \operatorname{Vinod~Kumar~}^{7}$, \\ Dinesh Kumar 1,*(D), Shashi Kant Bhatia $8, *$ (D) and Kamil Kuča ${ }^{4,9, *(D)}$
}

1 Food Technology Department, School of Bioengineering and Food Technology, Shoolini University of Biotechnology and Management Sciences, Solan 173229, Himachal Pradesh, India; microharshs@gmail.com

2 Botany Department, School of Biological and Environmental Sciences, Shoolini University of Biotechnology and Management Sciences, Solan 173229, Himachal Pradesh, India; kanchankannu1992@gmail.com (K.B.); rachnaverma@shooliniuniversity.com (R.V.)

3 Biotechnology Department, School of Bioengineering and Biosciences, Lovely Professional University, Phagwara 144411, Punjab, India; daljeetdhanja192@gmail.com (D.S.D.); reena.19408@lpu.co.in (R.S.)

4 Department of Chemistry, Faculty of Science, University of Hradec Kralove, 50003 Hradec Kralove, Czech Republic; eugenie.nepovimova@uhk.cz

5 Sen Research Group, Biochemistry Department, Faculty of Arts and Science, Dumlupınar University, Evliya Çelebi Campus, 43100 Kütahya, Turkey; fatihsen1980@gmail.com

6 Biotechnology Department, Applied Sciences and Biotechnology, Shoolini University of Biotechnology and Management Sciences, Solan 173229, Himachal Pradesh, India; haileregg@gmail.com

7 School of Water, Energy and Environment, Cranfield University, Cranfield MK430AL, UK; Vinod.Kumar@cranfield.ac.uk

8 Department of Biological Engineering, College of Engineering, Konkuk University, Seoul 05029, Korea

9 Biomedical Research Center, University Hospital Hradec Kralove, 50005 Hradec Kralove, Czech Republic

* Correspondence: dineshkumar@shooliniuniversity.com (D.K.); shashikonkukuni@konkuk.ac.kr (S.K.B.); kamil.kuca@uhk.cz (K.K.); Tel.: +420-603-289-166 (K.K.)

Received: 8 October 2020; Accepted: 9 November 2020; Published: 11 November 2020

\begin{abstract}
Fruit extracts have natural bioactive molecules that are known to possess significant therapeutic potential. Traditionally, metallic nanoparticles were synthesized via chemical methods, in which the chemical act as the reducing agent. Later, these traditional metallic nanoparticles emerged as the biological risk, which prompted researchers to explore an eco-friendly approach. There are different eco-friendly methods employed for synthesizing these metallic nanoparticles via the usage of microbes and plants, primarily via fruit extract. These explorations have paved the way for using fruit extracts for developing nanoparticles, as they eliminate the usage of reducing and stabilizing agents. Metallic nanoparticles have gained significant attention, and are used for diverse biological applications. The present review discusses the potential activities of phytochemicals, and it intends to summarize the different metallic nanoparticles synthesized using fruit extracts and their associated pharmacological activities like anti-cancerous, antimicrobial, antioxidant and catalytic efficiency.
\end{abstract}

Keywords: anticancer; antimicrobial; antioxidant; bioactive molecules; catalytic; fruits; metallic nanoparticles

\section{Introduction}

Nanotechnology has emerged as the revolutionary discipline of science with diverse application in the field of agriculture, biomedicine, catalysis, cosmetics, energy, electronics, mechanics, optics, 
pharmaceutics, sensors and textile [1]. These diverse applications have led to the advent of advancing discipline like nanobiotechnology [2]. Substantial growth in this field has inclined the interest of researchers to synthesize green nanoparticles (NPs) using different parts of plants. The primary reason for synthesizing metallic nanoparticles from different parts of the plants is that the procedures are cost-effective, eco-friendly, sustainable and straightforward [1,3]. Metal and metal oxide NPs are considered as efficient nanoparticles as they show remarkable biomedical activity and have a high surface area to volume ratio [1]. Predominantly, the bottom-up approach is used for synthesizing biogenic NPs, where atoms and compounds serve as the building block and self-assemble themselves to construct a final product [4]. In this, the biological system performs the function of biological laboratories for synthesizing pure metal as well as metal oxide nanoparticles via a biomimetic approach [5].

Lately, numerous herbal species and plant extracts have been used as capping and reducing agent for synthesizing NPs, which has improved the field of nanoscience [1]. Various bacterial cells, as well as their extracts, have been comprehended for synthesizing varieties of NPs of silver (Ag), gold (Au), silver oxide $(\mathrm{AgO})$, cadmium sulfide $(\mathrm{CdS})$, and titanium dioxide $\left(\mathrm{TiO}_{2}\right)$ [3]. A few fungal species have also been reported for synthesizing NPs of $\mathrm{Ag}, \mathrm{CdS}$ and $\mathrm{TiO}_{2}$. Other biological materials like honey have also been used for NPs from Ag, Au, carbon, lead (Pd) and platinum (Pt) [3]. On extensive analysis, plant-based NPs are more mono-dispersed and stable in contrast to microbial NPs.

Additionally, plants extracts have added advantage as they take less time to reduce the metal ions. The main reason for considering the natural plant-based extracts for synthesizing NPs is the use of green chemistry in their synthesis. The primary benefit of using green chemistry for synthesizing NPs is that it allows the selection of an eco-friendly reducing agent, solvent medium and non-toxic material for stabilization of NPs [3]. Diverse compounds like alkaloids, amines, amides, flavanones, terpenoids, proteins, phenolics and pigments are present in the plant extract, which aid in reduction and stabilization of metal ions during green NP synthesis [6]. Moreover, compounds like sugars, vitamins, peptides and water from tea and coffee extracts have been effectively used for synthesizing NPs [7-12]. Therefore, the current review intends to highlight the use of fruit extracts for synthesizing green metallic NPs. The first portion of the review discusses the role of fruits in human life and the second portion of the review emphasizes the different metallic NPs obtained through various fruit extracts as well as their applications.

\section{Health Benefits of Fruit Phytochemicals}

The ingestion of fruit and its products not only improves the health of the individual but also reduces the chances of different diseases like age-related macular degeneration, ageing, cardiovascular disease, cancer, cataract of the eye, compromised immune system, gastrointestinal disorder, hypertension, high cholesterol and lowering of low-density lipoprotein (LDL) [13]. To endorse a healthy lifestyle, USDA (United States Department of Agriculture) has recommended filling half of the plate with vegetables and fruits. This is because they have a substantial amount of dietary fibre, minerals (calcium, iron, magnesium and potassium), vitamins (ascorbic acid, folic acid, as well as vitamin A precursors) and various beneficial phytochemicals with antioxidant properties. Lately, FAO/WHO (Food and Agriculture Organization of the United Nations and World Health Organization) have stated to consume minimum $400 \mathrm{~g}$ of vegetable and fruits daily (exclusive of potatoes as well as other starchy tubers) to prevent chronic diseases (cancer, diabetes, heart disease and obesity) and alleviate the level of deficient micronutrients [14]. Origin and temperature of production area are the key factors which further enable the classification of fruits into tropical fruits, sub-tropical fruits and temperate fruits [13]. The NPs obtained from fruits has been known to possess various biological activities, as depicted in Figure 1. 


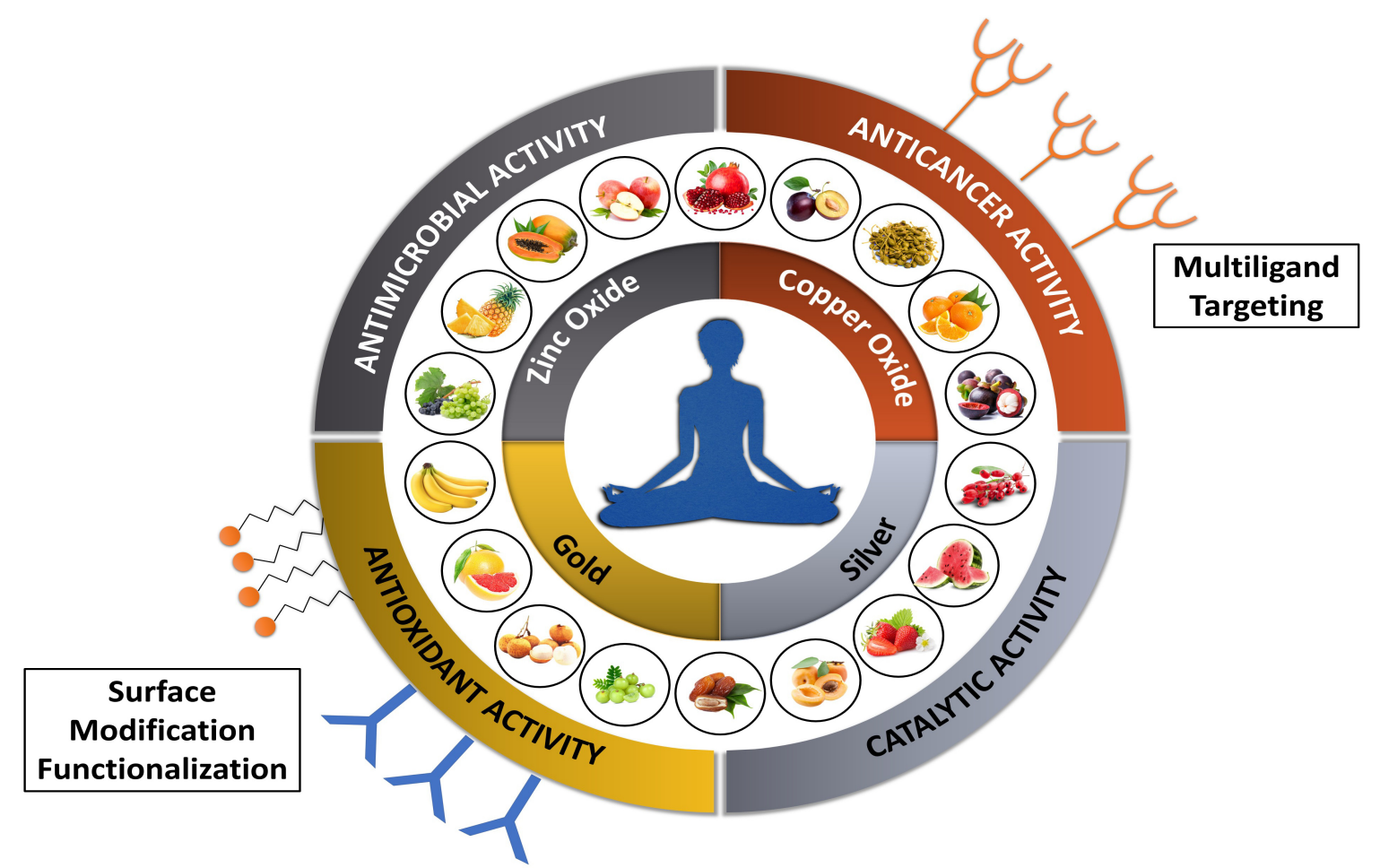

Figure 1. Graphical representation of fruit sources used for synthesizing nanoparticles with potential biological activities.

Carotenoids are the plant pigments that provide the red, yellow and orange colour to fruits. Approximately 600 carotenoids have been identified to date, out of which, around 50 get transformed into vitamin A [15]. Other than this, carotenoids are also known for their antioxidant potential and claimed for reducing the risk of diseases like cancer, cataracts, cardiovascular and macular degeneration. Additionally, they have a substantial role in improving the immune system [16,17]. Orange coloured fruits are the primary reservoir of $\beta$-carotene that are known for exhibiting highest provitamin A activity. Red carotenoid, i.e., lycopene, is predominantly found in pink grapefruit, tomato, watermelon and other red-coloured fruits and has been acclaimed as the valuable antioxidant in carotenoids family. Various animal, as well as human studies, have revealed that lycopene shows defending nature against different carcinogens of breast, brain, cervix, colon and prostate [18-21].

Furthermore, flavonoids are a group of phenolic compounds encompassing anthocyanins, flavanones, catechins, flavonols, flavones and isoflavones. To date, approximately 4000 flavonoids have been identified, and these flavonoids are dominantly found in citrus fruits and berries [15]. Published literature related to flavonoids has revealed that they have a myriad of benefits on humans like the potential to prevent cardiovascular disorders, cancers, urinary tract infections (UTIs) and other degenerative diseases [22-24]. Flavonoid Anthocyanins are blue pigments in blueberry and red pigments in strawberry and cherry. In addition to that, anthocyanins have been claimed to show to antioxidant potential in a biological system. Blackberry, Blueberry, Black raspberry and cranberry contain flavonoid proanthocyanidins, which have a substantial role in reducing the chances of UTIs [15]. The different phytochemicals obtained from fruits are used as a capping agent in NPs synthesis, and their biological applications in humans have been illustrated in Table 1. 
Table 1. Enlist of phytochemicals obtained from fruits with their role as a capping agent in metallic nanoparticles for human benefits.

\begin{tabular}{|c|c|c|c|c|c|}
\hline Fruit Verities & Phytochemicals & Role & $\begin{array}{c}\text { Types of } \\
\text { Metallic } \\
\text { Nanoparticles }\end{array}$ & $\begin{array}{l}\text { Phytochemicals as } \\
\text { Capping Agents }\end{array}$ & References \\
\hline $\begin{array}{l}\text { Banana, Amla, } \\
\text { Pomegranate }\end{array}$ & $\begin{array}{c}\text { Trans- } \beta \text { carotene, } \\
\beta \text {-Sitosterol, Caffeic acid, } \\
\text { Gallagic acid }\end{array}$ & Anticancer & Copper oxide & $\begin{array}{l}\text { Phenols, Primary } \\
\text { amines, Polyphenols, } \\
\text { Sterols, Fatty acids, } \\
\text { Hydroxyl, Carbonyl, } \\
\text { Terpenoids, Proteins }\end{array}$ & {$[13,25-32]$} \\
\hline $\begin{array}{l}\text { Banana, Amla, } \\
\text { Pomegranate, } \\
\text { Guava, Citron }\end{array}$ & $\begin{array}{l}\text { p-Coumaric acid, Vitamin } \\
\text { C, Emblicanin-A, Catechin, } \\
\text { Guavin-B, } \beta \text {-Bisabolene }\end{array}$ & Antioxidants & Gold & $\begin{array}{c}\text { Carboxyl, Aliphatic } \\
\text { amines, Phenols, } \\
\text { Flavonoids, Terpenes, } \\
\text { Vitamins, Lycopene, } \\
\text { Glycosides, Amino acids }\end{array}$ & $\begin{array}{c}{[13,25,26,} \\
33-40]\end{array}$ \\
\hline $\begin{array}{l}\text { Banana, Amla, } \\
\text { Guava, Citron }\end{array}$ & $\begin{array}{c}\text { Ferulic acid, } \\
\text { 1,6-bis-O-galloyl-beta- } \\
\text { D-glucose, Gallic acid, } \\
\text { Avicularin, Citral B, } \\
\text { Limonene, }\end{array}$ & Antimicrobial & Silver & $\begin{array}{c}\text { Phenols, Alkaloids, } \\
\text { Vitamins, Polyphenols, } \\
\text { Amino acids, } \\
\text { Carbohydrates, Proteins, } \\
\text { Flavonoids }\end{array}$ & $\begin{array}{l}{[13,25,33,34,} \\
\quad 41-47]\end{array}$ \\
\hline $\begin{array}{l}\text { Pomegranate, } \\
\text { Lemon, Grape, } \\
\text { Pineapple, Jamun }\end{array}$ & $\begin{array}{c}\text { Punicic acid, B-pinene, } \\
\text { Stilbenoid, Malic acid, } \\
\text { Bromelain }\end{array}$ & Skincare & Zinc oxide & $\begin{array}{l}\text { Phenols, Flavonoids, } \\
\text { Xanthones, Anthocyanin }\end{array}$ & {$[26,48-52]$} \\
\hline
\end{tabular}

\section{Green Synthesis of Nanoparticles Mediated by Fruit Extracts}

Extracts of fruits have been comprehended to contain a high amount of reducing agents. For example, fruits like blueberries, blackberries, Cornus mas L., Citrullus lanatus, grape, Terminalia arjuna and Punica granatum L., comprise a high number of anthocyanins, ascorbic acid, phenolic compounds, flavonoids, saccharides and other vitamins [53]. The synthesis of NPs from fruits has an additional advantage in comparison to NPs synthesized by the biological method. The biological method for NP synthesis is mediated by microbes, and microbes need to be pure strains and ought to be maintained in an aseptic environment. Moreover, the separation of nanoparticles from microbial broth culture during downstream processing is quite tricky. Furthermore, it takes time to transform the metallic salts (soluble) into the elemental oxide/elemental NPs [3]. A general mechanism involved in the biosynthesis of diverse nanoparticles using fruit extracts has been illustrated in Figure 2.

The diverse types of nanoparticles prepared using different fruits extracts have been discussed below:

The data related to the synthesis of NPs using different varieties have been comprehended in Table 2. 


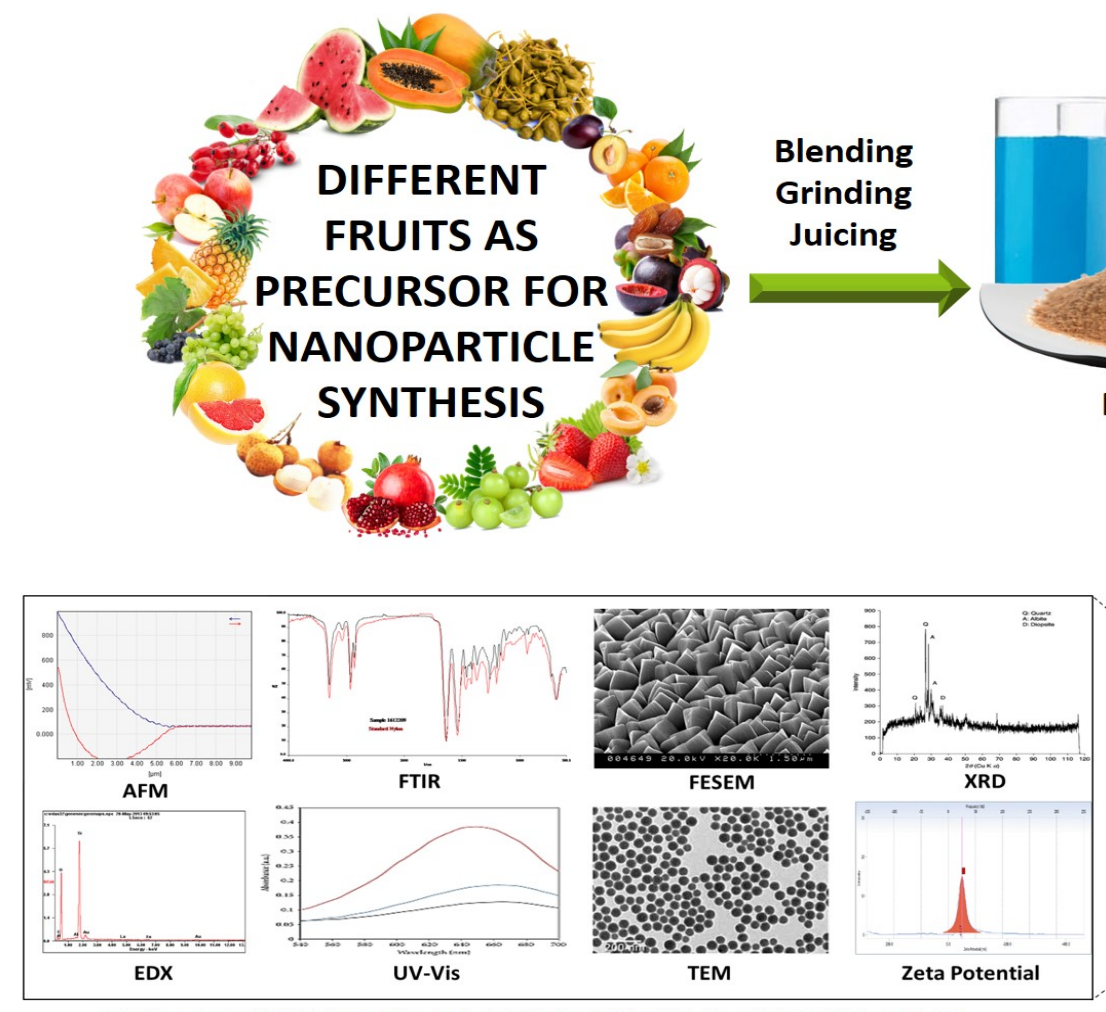

CHARACTERIZATION OF SYNTHESIZED NANOPARTICLES

Figure 2. A general mechanism of nanoparticle formation from fruit extracts. 
Table 2. Synthesis of nanoparticles (NPs) from various fruit verities.

\begin{tabular}{|c|c|c|c|c|c|c|c|c|}
\hline $\begin{array}{c}\text { Fruit Common } \\
\text { Name }\end{array}$ & Scientific Name & $\begin{array}{c}\text { Biological } \\
\text { Extract }\end{array}$ & $\begin{array}{l}\text { Types of NPs } \\
\text { synthesized }\end{array}$ & $\begin{array}{c}\text { Reaction } \\
\text { Temperature/Time }\end{array}$ & Morphology & Size & Stability & References \\
\hline North Arcot & $\begin{array}{l}\text { Syzygium alternifolium } \\
\text { (Wt.) Walp }\end{array}$ & Whole fruit & Copper oxide & $50^{\circ} \mathrm{C} / 2 \mathrm{~h}$ & Sphere & $2-69 \mathrm{~nm}$ & $\mathrm{Nd}$ & [27] \\
\hline $\begin{array}{l}\text { Christ's thorn } \\
\text { jujube }\end{array}$ & $\begin{array}{l}\text { Ziziphus spina-christi } \\
\text { (L.)Willd }\end{array}$ & Pulp & Copper oxide & $80^{\circ} \mathrm{C} / \mathrm{ND}$ & Sphere & $5-20 \mathrm{~nm}$ & $\mathrm{Nd}$ & [28] \\
\hline Caperberry & Capparis spinosa & Whole fruit & Copper oxide & $60^{\circ} \mathrm{C} / 24 \mathrm{~h}$ & Sphere & $17-41 \mathrm{~nm}$ & $\mathrm{Nd}$ & [29] \\
\hline Citron & Citrus medica Linn. & Juice & Copper oxide & $60-100^{\circ} \mathrm{C} / \mathrm{ND}$ & ND & $10-60 \mathrm{~nm}$ & $\mathrm{Nd}$ & [54] \\
\hline Strawberry & Fragaria ananassa & Whole fruit & Copper oxide & $\mathrm{RT} / 1 \mathrm{~h}$ & Sphere & $10-30 \mathrm{~nm}$ & $\mathrm{Nd}$ & [30] \\
\hline Guava & Psidium guajava $\mathrm{L}$ & Whole fruit & Copper oxide & $80^{\circ} \mathrm{C} / 2 \mathrm{~h}$ & Flakes & $15-30 \mathrm{~nm}$ & 15 days & [31] \\
\hline Pomegranate & Punica granatum & Peel & Copper oxide & $80^{\circ} \mathrm{C} / 10 \mathrm{~min}$ & Sphere & $15-20 \mathrm{~nm}$ & $\mathrm{Nd}$ & [32] \\
\hline Pomegranate & Punica granatum & Juice & Gold & RT/ND & $\begin{array}{l}\text { Triangular, Pentagonal, } \\
\text { Hexagonal and Sphere }\end{array}$ & $23-36 \mathrm{~nm}$ & $\mathrm{Nd}$ & [35] \\
\hline Wild orange & Citrus macroptera & Juice & Gold & $40-50^{\circ} \mathrm{C} / 90 \mathrm{~min}$ & Sphere & $7-25 \mathrm{~nm}$ & $\mathrm{Nd}$ & [55] \\
\hline Longan & Euphoria longana Lam. & Juice & Gold & $30^{\circ} \mathrm{C} / 30 \mathrm{~min}$ & Sphere & $25 \mathrm{~nm}$ & $\mathrm{Nd}$ & [36] \\
\hline Pomegranate & Punica granatum & Juice & Gold & $\mathrm{RT} / 20 \mathrm{~min}$ & Irregular & $100 \mathrm{~nm}$ & $\mathrm{Nd}$ & [37] \\
\hline Pomelo & Citrus maxima & Juice & Gold & $\mathrm{RT} / 5 \mathrm{~min}$ & Rod and Sphere & $25.7 \mathrm{~nm}$ & $\mathrm{Nd}$ & [38] \\
\hline Watermelon & Citrullus lanatus & Rind & Gold & $\mathrm{RT} / 1 \mathrm{~h}$ & Sphere & $20-140 \mathrm{~nm}$ & 1 month & [39] \\
\hline Plum & Prunus domestica & Whole fruit & Gold & $\mathrm{RT} / 4 \mathrm{~h}$ & Sphere & $4-38 \mathrm{~nm}$ & $\mathrm{Nd}$ & [40] \\
\hline Pomegranate & Punica granatum & Juice & Silver & $65^{\circ} \mathrm{C} / 1 \mathrm{~min}$ & Cubic & $23 \mathrm{~nm}$ & $\mathrm{Nd}$ & [56] \\
\hline Papaya & Carica papaya & Juice & Silver & NS & Sphere & $75.68 \mathrm{~nm}$ & $\mathrm{Nd}$ & [41] \\
\hline Chebulic myrobalan & Terminalia chebula & Whole fruit & Silver & $\mathrm{RT} / \mathrm{ND}$ & Cubic & $25 \mathrm{~nm}$ & $\mathrm{Nd}$ & [42] \\
\hline Grape & Vitis vinifera & Whole fruit & Silver & $\mathrm{RT} / 4 \mathrm{~h}$ & Sphere & $30-40 \mathrm{~nm}$ & $\mathrm{Nd}$ & [57] \\
\hline Indian gooseberry & Emblica officinalis & Pulp & Silver & $\mathrm{RT} / 30 \mathrm{~min}$ & Sphere & $15 \mathrm{~nm}$ & $\mathrm{Nd}$ & [43] \\
\hline Indian gooseberry & Phyllanthus emblica & Pulp & Silver & $65^{\circ} \mathrm{C} / 20 \mathrm{~min}$ & Sphere & $19.8-92.8 \mathrm{~nm}$ & $\mathrm{Nd}$ & [44] \\
\hline Fig & Ficus carica & Whole fruit & Silver & $\mathrm{RT} / 24 \mathrm{~h}$ & Sphere & $54-89 \mathrm{~nm}$ & $\mathrm{Nd}$ & [45] \\
\hline Indian gooseberry & Phyllanthus emblica & Pulp & Silver & RT/ND & Cubic & $19-45 \mathrm{~nm}$ & $\mathrm{Nd}$ & [46] \\
\hline Black hawthorn & Crataegus pentagyna & Pulp & Silver & $\mathrm{RT} / 2 \mathrm{~h}$ & Sphere & $25-45 \mathrm{~nm}$ & $\mathrm{Nd}$ & [47] \\
\hline Date palm & Phoenix dactylifera & Pulp & Silver & $60^{\circ} \mathrm{C} / 20 \mathrm{~min}$ & Sphere & $20-100 \mathrm{~nm}$ & $\mathrm{Nd}$ & [58] \\
\hline Date palm & Phoenix dactylifera & Pulp & Silver & $55^{\circ} \mathrm{C} / 10 \mathrm{~min}$ & Sphere & $25-60 \mathrm{~nm}$ & $\mathrm{Nd}$ & [59] \\
\hline Apple & Malus pumila & Pulp & Silver & $80^{\circ} \mathrm{C} / \mathrm{ND}$ & Sphere & $30.25 \mathrm{~nm}$ & $\mathrm{Nd}$ & [60] \\
\hline Pomegranate & Punica granatum & Peel & Silver & $\mathrm{RT} / 24 \mathrm{~h}$ & ND & $5-50 \mathrm{~nm}$ & $\mathrm{Nd}$ & [61] \\
\hline Banana & Musa paradisiaca & Peel & Silver & $30^{\circ} \mathrm{C} / \mathrm{ND}$ & Sphere & $23.7 \mathrm{~nm}$ & $\mathrm{Nd}$ & [62] \\
\hline Banana & Musa paradisiaca & Peel & Silver & $\mathrm{RT} / 30 \mathrm{~min}$ & Grain & $34 \mathrm{~nm}$ & $\mathrm{Nd}$ & [63] \\
\hline Orange & Citrus sinensis & Peel & Silver & $90^{\circ} \mathrm{C} / 15 \mathrm{~min}$ & Sphere & $7.36 \mathrm{~nm}$ & $\mathrm{Nd}$ & [64] \\
\hline Apricot & Prunus armeniaca & Peel & Silver & NS & Rod & $50 \mathrm{~nm}$ & $\mathrm{Nd}$ & [65] \\
\hline
\end{tabular}


Table 2. Cont

\begin{tabular}{|c|c|c|c|c|c|c|c|c|}
\hline $\begin{array}{c}\text { Fruit Common } \\
\text { Name }\end{array}$ & Scientific Name & $\begin{array}{c}\text { Biological } \\
\text { Extract }\end{array}$ & $\begin{array}{l}\text { Types of NPs } \\
\text { synthesized }\end{array}$ & $\begin{array}{c}\text { Reaction } \\
\text { Temperature/Time }\end{array}$ & Morphology & Size & Stability & References \\
\hline Pomegranate & Punica granatum & Peel & Silver & $\mathrm{RT} / 24 \mathrm{~h}$ & Sphere & $20-40 \mathrm{~nm}$ & $\mathrm{Nd}$ & [66] \\
\hline Pineapple & Ananas comosus & Peel & Silver & $\mathrm{RT} / 24 \mathrm{~h}$ & Sphere & ND & $\mathrm{Nd}$ & {$[67]$} \\
\hline Logan & Dimocarpus Longan Lour & Peel & Silver & $80^{\circ} \mathrm{C} / 5 \mathrm{~h}$ & Cubic & $9-32 \mathrm{~nm}$ & 6 months & [68] \\
\hline Pomelo & Citrus maxima & Juice & Zinc oxide & $400^{\circ} \mathrm{C} / 5-10 \mathrm{~min}$ & Agglomerated & $10-20 \mathrm{~nm}$ & $\mathrm{Nd}$ & [69] \\
\hline Purple mangosteen & Garcinia mangostana & Pulp & Zinc oxide & $70-80^{\circ} \mathrm{C} / \mathrm{ND}$ & Sphere & $21 \mathrm{~nm}$ & $\mathrm{Nd}$ & {$[52]$} \\
\hline Pomegranate & Punica granatum & Peel & Zinc oxide & $80^{\circ} \mathrm{C} / \mathrm{ND}$ & Sphere and Hexagonal & $32-81 \mathrm{~nm}$ & $\mathrm{Nd}$ & {$[70]$} \\
\hline Pineapple & Ananas comosus & Juice & Zinc oxide & $240^{\circ} \mathrm{C} / 5 \mathrm{~min}$ & ND & $30-57 \mathrm{~nm}$ & $\mathrm{Nd}$ & [71] \\
\hline
\end{tabular}




\subsection{Copper Oxide Nanoparticles ( $\left.\mathrm{Cu}_{2} \mathrm{ONPs}\right)$}

Copper oxide $\left(\mathrm{Cu}_{2} \mathrm{O}\right)$ is claimed to be a transition metal oxide having narrow bandgap, i.e., $\sim 2.0 \mathrm{eV}$ and shows distinct features like significant electrochemical activity, improved redox potential, high specific surface area and incomparable stability in solutions. It is the second choice of researchers working in the field of nanotechnology after the noble metal nanoparticles, owing to its propitious application in different subjects like antifouling coatings, biocidal agents, catalysis, sensors/biosensors, electrochemistry and energy storage [72]. These nanoparticles are extensively used for non-enzymatic sensing of clinical analytes due to their ability to promote electron transfer reaction even at low potential [72]. The $\mathrm{Cu}_{2} \mathrm{ONPs}$ derived from fruits have been listed along with various applications in Table 3.

Table 3. Applications of copper oxide NPs synthesized from various fruit varieties.

\begin{tabular}{|c|c|c|c|}
\hline Family & Fruit Verity & Applications & References \\
\hline Myrtaceae & $\begin{array}{l}\text { Syzygium alternifolium } \\
\text { (Wt.) Walp. }\end{array}$ & Antiviral activity against Newcastle Disease Virus (NDV) & [27] \\
\hline Rhamnaceae & $\begin{array}{l}\text { Ziziphus spina-christi } \\
\text { (L.)Willd }\end{array}$ & $\begin{array}{l}\text { Adequate adsorption capacity to the removal of crystal } \\
\text { violet (CV), from aqueous solution; Antibacterial activity } \\
\text { against Escherichia coli and Staphylococcus aureus }\end{array}$ & [28] \\
\hline Capparaceae & Capparis spinosa & Antibacterial activity against $S$. aureus, Bacillus cereus & [29] \\
\hline Rutaceae & Citrus medica Linn. & $\begin{array}{l}\text { Antibacterial activity against E. coli, Klebsiella pneumoniae, } \\
\text { Pseudomonas aeruginosa, Propionibacterium acnes and } \\
\text { Salmonella typhi; Antifungal activity against Fusarium } \\
\text { culmorum, F. oxysporum and F. graminearum }\end{array}$ & [54] \\
\hline Rosaceae & Fragaria ananassa & $\begin{array}{l}\text { Antibacterial activity against S. aureus, S. saprophyticus, } \\
\text { Bacillus subtilis, Streptococcus pneumoniae, E. coli O157: H7, S. } \\
\text { typhimurium, Proteus mirabilis, and P. aeruginosa; Antifungal } \\
\text { activity against Candida guilliermondii, C. parapsilosis, C. } \\
\text { albicans, C. krusei, and C. glabrata; Antioxidant activity; } \\
\text { Cutaneous wound healing ability }\end{array}$ & [30] \\
\hline Myrtaceae & Psidium guajava $\mathrm{L}$ & Antibacterial activity against $E$. coli and $S$. aureus & [31] \\
\hline Lythraceae & Punica granatum & $\begin{array}{l}\text { Antibacterial activity against Micrococcus luteus MTCC } 1809 \text {, } \\
\text { P. aeruginosa MTCC } 424 \text {, Salmonella enterica MTCC } 1253 \text { and } \\
\text { Enterobacter aerogenes MTCC } 2823\end{array}$ & [32] \\
\hline
\end{tabular}

\subsection{Gold Nanoparticles (AuNPs)}

In recent years, Gold nanoparticles (AuNPs) have gained substantial attention owing to their biocompatibility, optical and physical (shape and size) properties [73]. AuNPs of diverse morphology and varied size are extensively employed in medicine for different purposes such as for the detection of tumours, as a drug carrier, etc. [73]. The AuNPs derived from fruits has been listed along with various applications in Table 4.

Table 4. Applications of gold NPs synthesized from various fruit varieties.

\begin{tabular}{|c|c|c|c|}
\hline Family & Fruit Verity & Applications & References \\
\hline Lythraceae & Punica granatum & Catalytic activity against 4-nitrophenol & [35] \\
\hline Rutaceae & Citrus macroptera & $\begin{array}{l}\text { Antibiofilm activity against Pseudomonas aeruginosa; } \\
\text { Cytotoxic effect against HepG2 (liver cancer cell line) }\end{array}$ & [55] \\
\hline Sapindaceae & Euphoria longana Lam. & $\begin{array}{l}\text { Cytotoxicity against human breast cancer cell lines MCF-7; } \\
\text { Antioxidant activity }\end{array}$ & [36] \\
\hline Lythraceae & Punica granatum & Antioxidant activity & [37] \\
\hline Rutaceae & Citrus maxima & $\begin{array}{l}\text { Catalytic activity against 4-nitrophenol } \\
\text { Antibacterial activity against Bacillus cereus ATCC 13061, } \\
\text { Escherichia coli ATCC 43890, Listeria monocytogenes ATCC }\end{array}$ & [38] \\
\hline Cucurbitaceae & Citrullus lanatus & $\begin{array}{l}\text { 19115, Staphylococcus aureus ATCC } 49444 \text {, Salmonella } \\
\text { typhimurium ATCC } 43174 ; \text { Antioxidant activity; } \\
\text { Anti-proteasome inhibitory potential }\end{array}$ & [39] \\
\hline Rosaceae & Prunus domestica & Catalytic activity against 4-nitrophenol & {$[40]$} \\
\hline Vitaceae & Vtis vinifera & $\begin{array}{l}\text { Apoptotic activity against human epidermoid carcinoma } \\
\text { A431 cell line }\end{array}$ & {$[74,75]$} \\
\hline
\end{tabular}




\subsection{Silver Nanoparticles (AgNPs)}

Silver nanoparticles (AgNPs) have also gained significant attention due to their biochemical and catalytic activity owing to their large surface area in contrast to other particles with analogous chemical structures [73]. Synthesis of AgNPs occurs in two steps: in the first step, $\mathrm{Ag}^{+}$ions are reduced to $\mathrm{Ag}^{\circ}$, and in the second step, clustering of colloidal AgNPs take place to form oligomeric clusters which finally gets stabilized [73]. The reduction of $\mathrm{Ag}^{+}$ions requires biological catalysts, i.e., enzymes, which are obtained from different biological sources like microbes, fruits extracts, plants, etc. Moreover, a variety of fruits extracts have already been comprehended for synthesizing AgNPs with diverse biological potential, as listed in Table 5 .

Table 5. Applications of silver NPs synthesized from various fruit varieties.

\begin{tabular}{|c|c|c|c|}
\hline Family & Fruit Verity & Applications & References \\
\hline Combretaceae & Terminalia chebula & Catalytic activity against methylene blue & [42] \\
\hline Vitaceae & Vitis vinifera & $\begin{array}{l}\text { Antibacterial activity against Bacillus subtilis and } \\
\text { Klebsiella planticola }\end{array}$ & [57] \\
\hline Phyllanthaceae & Emblica officinalis & $\begin{array}{l}\text { Antibacterial activity against Staphylococcus aureus, } \\
\text { B. subtilis, Escherichia coli and Klebsiella pneumoniae }\end{array}$ & [43] \\
\hline Phyllanthaceae & Phyllanthus emblica & Antibacterial activity against Acidovorax oryzae strain RS-2 & [44] \\
\hline Moraceae & Ficus carica & Cytotoxicity against human breast cancer cell lines MCF-7 & [45] \\
\hline Phyllanthaceae & Phyllanthus emblica & Antibacterial activity against $S$. aureus, K. pneumoniae & [46] \\
\hline Rosaceae & Crataegus pentagyna & $\begin{array}{l}\text { Antibacterial activity against } E \text {. coli, S. aureus, Enterococcus } \\
\text { faecalis, Pseudomonas aeruginosa, Acinetobacter baumannii; } \\
\text { Photocatalytic action against rhodamine b, eosin and } \\
\text { methylene blue }\end{array}$ & [47] \\
\hline Arecaceae & Phoenix dactylifera & $\begin{array}{l}\text { Antibacterial activity against } E \text {. coli, } S \text {. aureus, E. faecalis, } P \text {. } \\
\text { aeruginosa; Antifungal activity against Candida albicans; } \\
\text { Cytotoxicity against human breast cancer cell lines MCF-7 }\end{array}$ & [58] \\
\hline Arecaceae & Phoenix dactylifera & $\begin{array}{l}\text { Antibacterial activity against } S \text {. aureus, } S \text {. epidermidis, } K . \\
\text { pneumoniae, and E. coli; Catalytic activity against } \\
\text { 4-nitrophenol }\end{array}$ & [59] \\
\hline Rosaceae & Malus pumila & $\begin{array}{l}\text { Antibacterial activity against E. coli, S. aureus, P. aeruginosa } \\
\text { and methicillin-resistant } S \text {. aureus }\end{array}$ & [60] \\
\hline Lythraceae & Punica granatum & Antibacterial activity against $E$. coli, S. aureus, P. aeruginosa & [61] \\
\hline Musaceae & Musa paradisiaca & $\begin{array}{l}\text { Antibacterial activity against } E \text {. coli, } S \text {. aureus, P. aeruginosa, } \\
\text { B. subtilis; Antifungal activity against Candida albicans }\end{array}$ & [62] \\
\hline Musaceae & Musa paradisiaca & $\begin{array}{l}\text { Antibacterial activity against E. coli, K. pneumoniae, S. } \\
\text { aureus, B. subtilis; Antioxidant activity }\end{array}$ & [63] \\
\hline Rosaceae & Prunus armeniaca & $\begin{array}{l}\text { Antibacterial activity against E. coli, S. aureus, P. aeruginosa, } \\
\text { B. subtilis }\end{array}$ & [65] \\
\hline Lythraceae & Punica granatum & $\begin{array}{l}\text { Antibacterial activity against } E \text {. coli, } S \text {. aureus, } S \text {. } \\
\text { epidermidis, } P \text {. aeruginosa, Proteus vulgaris, Salmonella typhi, } \\
\text { K. pneumoniae; Cytotoxicity against colon cancer cell line } \\
\text { (RKO: ATCC }{ }^{\circledR} \text { CRL-2577 }{ }^{\mathrm{TM}} \text { ) }\end{array}$ & [66] \\
\hline Bromeliaceae & Ananas comosus & $\begin{array}{l}\text { Antioxidant activity; Cytotoxic effect against HepG2 (liver } \\
\text { cancer cell line); Anti-diabetic activity; Antibacterial } \\
\text { activity against Bacillus cereus KCTC } 3624 \text {, Listeria } \\
\text { monocytogenes ATCC } 19111 \text {, Enterococcus faecium DB01, } \\
\text { and S. aureus ATCC } 13565\end{array}$ & [67] \\
\hline Sapindaceae & $\begin{array}{l}\text { Dimocarpus Longan } \\
\text { Lour }\end{array}$ & $\begin{array}{l}\text { Antibacterial activity against E. coli, S. aureus, P. aeruginosa, } \\
\text { B. subtilis; Antifungal activity against Candida albicans; } \\
\text { Cytotoxicity against PC-3 (prostate cancer cell line) }\end{array}$ & [68] \\
\hline
\end{tabular}

\subsection{Zinc Oxide Nanoparticles (ZnONPs)}

Lately, ZnONPs has intrigued researchers working in the field of nanotechnology owing to their diverse application in different areas such as biomedical, electronics and optical sector [76]. Synthesis of ZnONPs is considered to be cost-effective, easy and safe. Even, FDA has given the generally recognized as safe (GRAS) status to $\mathrm{ZnO}[77,78]$. ZnONPs have been primarily comprehended for anti-inflammatory properties and wound healing in the medical sector [76]. Nowadays, ZnONPs are 
predominantly used in cosmetic products like sunscreen lotions, as they exhibit intrinsic UV filtering potential [76]. Other than this, ZnONPs are used in drug delivery system as they show anti-cancerous, antifungal, antimicrobial and anti-diabetic properties [76]. The ZnONPs derived from fruits has been listed along with various applications in Table 6.

Table 6. Applications of zinc oxide NPs synthesized from various fruit varieties.

\begin{tabular}{|c|c|c|c|}
\hline Family & Fruit Verity & Applications & References \\
\hline Rutaceae & Citrus maxima & $\begin{array}{l}\text { Photocatalytic activity against methylene blue; } \\
\text { Antibacterial activity against Klebsiella aerogenes, } \\
\text { E. coli, S. aureus; Sensor activity towards dopamine }\end{array}$ & [69] \\
\hline Clusiaceae & Garcinia mangostana & Photocatalytic activity against malachite green & [52] \\
\hline Lythraceae & Punica granatum & $\begin{array}{l}\text { Antibacterial activity against Escherichia coli and } \\
\text { Enterococcus faecalis; Cytotoxicity against HCT116 } \\
\text { (colorectal cancer cell line) }\end{array}$ & {$[70]$} \\
\hline Bromeliaceae & Ananas comosus & Antibacterial activity against $E$. coli & [71] \\
\hline
\end{tabular}

\section{Anticancer Activity of Fruit-Derived NPs}

Cancer is one of the leading diseases, which has accounted for 9.6 million deaths worldwide in 2018, where 70\% of deaths occurred in low- and middle-income countries [79]. Chemotherapy, hormone therapy, radiation therapy and surgery are the primary approaches applied for management and treatment of cancer. Various medicinal plants having anti-cancerous and cytotoxic potential have already been recorded [80]. Polyphenols like alkaloids, flavonoids, phenolic acids and terpenes have been claimed to be responsible for the biological activity of plants [81-83]. Triterpenoids like avicins, boswellic acids, fomitellic acids, oleanolic acid, pomolic acid and ursolic acid have been comprehended for exhibiting cytotoxic effects [84]. Flavonoids like rutin, myricetin, kaempferol and quercetin have also been reported to show anticancer potential [82]. Application of nanotechnology in combating cancer has unfolded the new avenues for interdisciplinary research involving the different fields like biology, chemistry, engineering and medicine for diagnosis, detection and treatment [85]. In recent times, Myocet ${ }^{\mathrm{TM}}$ (Perrigo, Dublin, Ireland), Doxil ${ }^{\circledR}$ (Johnson and Johnson, New Brunswick, NJ, USA) and Abraxane ${ }^{\circledR}$ (Celgene, Summit, NJ, USA), the nano-based anti-cancerous drugs have been approved for clinical use by Food and Drug Administration (FDA-USA) [86].

AuNPs have been prepared using Citrus macroptera (CM) juice and have been evaluated for anti-cancerous potential via in vitro analysis on HepG2 liver cancer cells [55]. The result obtained from the study showed the $\mathrm{IC}_{50}$ value of $70.2 \mathrm{ng} / \mathrm{mL}$ for CM-AuNPs. This study was conducted to unveil the real potential of $\mathrm{CM}$ as it is used in traditional medicine by Tripura tribal people for treating liver ailments. Khan et al. [36] developed AuNPs using longan fruit juice and evaluate for anti-cancerous activity on MCF-7 (human breast cancer cells). The result obtained for the study revealed that increasing the concentration of AuNPs to $6.25-100 \mu \mathrm{g} / \mathrm{mL}$ substantially decreases the viability of these cells [36]. Moreover, spherical morphology, small size $(25 \mathrm{~nm})$, capping of phytochemicals and uniform distribution are considered as critical factors to exhibit considerable anti-cancerous potential. Additionally, the capping phytochemicals also help in regulating the expression of pro-apoptotic Bax and anti-apoptotic Bcl-2 protein

Jacob et al. [45] developed AgNPs via dried fruit extract of Ficus carica and evaluated for cytotoxic potential against MCF-7 (human breast cancer cells). The result showed the $\mathrm{LD}_{50}$ of AgNPs was $12.411 \mu \mathrm{g}$ in contrast to fruit extract, which was $139.04 \mu \mathrm{g}$. The analysis of cytotoxic potential was done assessing the level of ROS. On the other hand, Phoenix dactylifera mediated AgNPs exhibited dose-dependent cytotoxicity against MCF-7 [58]. The result obtained from the study showed the highest inhibitory effect at a concentration of $200 \mu \mathrm{g} / \mathrm{mL}$. Ananas comosus (L.) peel extract mediated AgNPs have also been recorded for anti-cancerous activity against HepG2 cancer cell line at high concentrations [67]. AgNPs derived from peel extract of Punica granatum have been reported to show 55-62\% toxicity at a dosage of $12.5 \mu \mathrm{g}$ against cell line of colon cancer [66]. The study enlightens us about the programmed 
cell death through autophagy. AgNPs prepared using peel extract of Dimocarpus Longan Lour have also been reported to show dose-dependent cytotoxic effects against prostate cancer cell line (PC-3) [68]. The concentration of AgNPs in the range of $5-10 \mu \mathrm{g} / \mathrm{mL}$ was found to reduce the viability of PC-3 cells by $50 \%$. Moreover, the $\mathrm{IC}_{50}$ was also less than $10 \mu \mathrm{g} / \mathrm{mL}$ and cytotoxic potential of these NPs was confirmed by suppression in the expression of BCL-2, STAT3 and survivin, whereas upregulation of the expression of caspase-3. Furthermore, AuNPs derived using grape peel and seeds have been evaluated for cytotoxic potential. The action of AuNPs caused the increase in the level of ROS, stimulated apoptosis as well as apoptotic morphological changes in A431 cells. These changes were claimed to be associated with interference of AuNPs with mitochondrial membrane potential [74,75]. It was concluded in the study that the reduction in mitochondrial membrane potential triggers the apoptotic cascade reaction in AuNPs treated cells.

\section{Antimicrobial Activity of Fruit-Derived NPs}

NPs have the potential to show antimicrobial activity, as NPs pass via the membrane of bacteria which influence the cell activity along with metabolic pathways [87]. After entering and interfering with the metabolic pathway of the bacteria cell, NPs adhere themselves with elementary components such as DNA, enzymes, liposomes and ribosomes. The association of elementary components with NPs leads to heterogeneous alterations, oxidative stress and alterations in cell membrane permeability, deactivation of proteins, enzyme inhibition, imbalance of electrolytes and alterations in gene expression [87].

Cell membranes and walls serve as protective checkpoints and impart resistance to the bacteria from the external environment. The bacterial cell wall also plays a primary role in conferring shape to the bacteria. Diverse pathways are involved in the absorption of NPs via the cell membrane in both Gram-positive and Gram-negative bacteria [88]. In Gram-negative bacteria, lipopolysaccharides (LPS) offer a negative charge for attracting NPs. Whereas, teichoic acid is present in Gram-positive bacteria to perform a similar function. Hence, NPs circulate through the phosphate molecular chain and circumvent accumulation. Moreover, NPs are found to be effective against Gram-positive in contrast to Gram-negative, owing to the presence of lipoproteins, LPS and phospholipids, which acts as the barrier and allows the movement of macromolecules only. Whereas, cell death and damage to cell membrane takes place in Gram-positive bacteria as the cell wall comprises teichoic acid, a thin layer of peptidoglycan and has abundant pores which allow the entry of foreign molecules [87].

$\mathrm{Cu}_{2} \mathrm{ONPs}$ derived by using Ziziphus spina-christi (L.) extract has been reported for exhibiting antimicrobial activity against Staphylococcus aureus in contrast to Escherichia coli [28]. Another study reported about $\mathrm{Cu}_{2} \mathrm{ONPs}$ derived from fruit extract of Capparis spinosa, which showed resilient antimicrobial activity against Bacillus cereus and S. aureus in contrast to E. coli and Klebsiella pneumoniae [29]. Citrus macroptera fruit extract mediated AuNPs have also been reported for inhibiting the proliferation of biofilm-producing Pseudomonas aeruginosa. The result from the study revealed that $12 \mathrm{ng} / \mathrm{mL}$ concentration of particles was effective for inhibiting the $60 \%$ growth of the biofilm [55]. Extract of Emblica officinalis has been used for synthesizing AgNPs. The synthesized AgNPs have been reported to exhibit high antimicrobial potential in contrast to fruit extract E. officinalis alone [43]. Biological derived AgNPs have been recorded to have effective antimicrobial activity against Bacillus subtilis, E. coli, K. pneumoniae and S. aureus [43]. AgNPs derived using fruit extract of Crataegus pentagyna has been accorded with minimum inhibitory concentration and minimum bactericidal concentration of $0.11,0.22,0.11,0.44,0.11,1.7,0.11,0.22$ and $0.11,7.1 \mu \mathrm{g} / \mathrm{mL}$ against Acinetobacter baumannii, E. coli, Enterococcus faecalis, P. aeruginosa and S. aureus, respectively [47]. Furthermore, ZnONPs derived using an extract of Citrus maxima has been recorded to exhibit significant antimicrobial activity against pathogenic microbes like Klebsiella aerogenes and S. aureus, whereas, and less significant towards E. coli [69]. 


\section{Antioxidant Activity of Fruit-Derived NPs}

$\mathrm{Cu}_{2} \mathrm{ONPs}$ derived using strawberry has been reported to exhibit remarkable concentrationdependent DPPH radical scavenging activity [30]. The interaction of strawberry extract with both $\mathrm{Cu}_{2} \mathrm{ONPs}$ and DPPH occurs via the transfer of electrons as well as hydrogen ions to 2,2-diphenyl-1picrylhydrazyl radical to convert itself to a 2,2-diphenyl-1-picrylhydrazine molecule (DPPH). Usually, DPPH shows strong absorbance at a wavelength of $517 \mathrm{~nm}$. However, due to the gain of an electron/hydrogen atom from an antioxidant molecule, it transforms and forms a steady diamagnetic molecule which showed a reduction in absorbance at $517 \mathrm{~nm}$. Additionally, the transformation of colour from purple to pale yellow helps to determine the anti-radical potential of antioxidants [30]. Anthocyanins, flavanols, flavan-3-ols and tannins (ellagitannins and gallotannins) are some of the antioxidant molecules obtained from strawberry [30]. These compounds have been comprehended for maintaining the redox homeostasis via following multiple steps of antioxidant reactions which includes initiation, branching, propagation and termination steps of free radicals.

AuNPs derived from the juice of Longan fruit has been accorded to show dose-dependent antioxidant activity against DPPH, which increases with increase in AuNPs [36]. Gubitosa et al. [37] conducted the study to assess the antioxidant potential of AuNPs derived using Punica granatum juice on $\mathrm{H}_{2} \mathrm{O}_{2}$ (ROS model). Protein cytochrome-c is highly sensitive to $\mathrm{H}_{2} \mathrm{O}_{2}$, but oxidative degradation alters its catalytic potential. The result obtained from the study unveils that an increase in AuNPs significantly decreases the Cyt-c degradation rate. In simple words, the presence of AuNPs delays the Cyt-c degradation process. AgNPs synthesized by Cavendish banana peel extract (CBPE) has been evaluated for antioxidant potential against DPPH [63]. The result obtained revealed that the inhibition rate of CBPE-mediated AgNPs is 64\% which increases with an increase in AgNP concentration. Moreover, CBPE-mediated AgNPs have also been reported for scavenging 2,2'-azinobis(3-ethylbenzothiazoline-6-sulfonic acid) (ABTS).

\section{Catalytic Activity of Fruit-Derived NPs}

The compound 4-nitrophenol and its derivatives are predominantly found in synthetic dyes, herbicides and insecticides, which are chiefly organic pollutants accorded from harming ecosystems [89]. Due to the inhibitory and toxic nature of 4-nitrophenol, it is regarded as a massive risk to the ecosystem. Hence, the degradation of these pollutants has become a matter of utmost importance. Despite this, the reduced product of 4-nitrophenol is used as a mediator in black/white film developers, rubber antioxidants, sulfur dyes, paracetamol, corrosion inhibition and act as precursors in analgesic and antipyretic drugs $[90,91] . \mathrm{NaBH}_{4}$ is extensively used as a metal catalyst, and reductant for $\mathrm{Cu}_{2} \mathrm{ONPs}$, AgNPs, AuNPs and PdNPs; it is claimed to be the most effective method to reduce 4-nitrophenol [92-95]. Methylene blue (MB) is a heterocyclic aromatic pollutant released from the dying industries [96]. $\mathrm{NaBH}_{4}$ is used as a reducing agent for MB and NPs, which serves the purpose of an absorbent [97].

$\mathrm{Cu}_{2} \mathrm{ONPs}$ derived using fruit extract of Ziziphus spina-christi (L.) has been reported to absorb $95 \%$ of crystal violet under controlled conditions, i.e., $\mathrm{pH} 9$, the dye concentration of $35 \mu \mathrm{g} \mathrm{mL}^{-1}$, stirring time of $7.5 \mathrm{~min}$ and sorbent amount of $80 \mathrm{mg}$ [28]. Colloidal gold nanoparticles synthesized using juice extract of Punica granatum have been reported to reduce 4-nitrophenol to 4-aminophenol in $12 \mathrm{~min}$ [35]. Whereas, AuNPs derived using Citrus maxima took $22 \mathrm{~min}$ for the same [38]. Terminalia chebula derived AgNPs have been reported for maximum reduction of MB to leucomethylene blue (colourless) within 30 min via electron relay effect [42]. Ebrahimzadeh et al. [47] reported the degradation of organic dyes like eosin (EY), methylene blue (MB) and rhodamine $b(\mathrm{RhB})$ with degradation percentages of $70 \%, 78 \%$ and $85 \%$, respectively, by Crataegus pentagyna fruit extract-derived AgNPs within 90 min under sunlight. ZnONPs derived using fruit pericarp of Garcinia mangostana have been reported to degrade malachite green dye by $99 \%$ within 180 min under sunlight in aqueous solution due to effective oxidation via hydroxyl radicals $(\bullet \mathrm{OH})$ synthesized during photocatalytic reactions [52]. 


\section{Conclusions}

Nature has its unique way of creating highly efficient miniature functionalized materials. Increased responsiveness in the direction of green chemistry and its use in synthesizing metallic nanoparticles has generated an aspiration to develop eco-friendly approaches. The advantage of using fruit extracts for synthesizing nanoparticles is that they are cost-effective, economical, energy-efficient, safe, and environment-friendly, do not affect human health and produce less waste. These green synthesized nanoparticles are being evaluated in the field of nanotechnology for diverse applications. Moreover, the use of fruit extracts for synthesizing nanoparticles has the added advantage over other biological procedures, which are time-consuming and require the maintenance of microbial cultures to sustain the actual potency during the nanoparticle synthesis. Therefore, the usage of plant extracts for deriving nanoparticles can have a massive impact in the coming years. Several works can be cited for the synthesis of nanoparticles via fruit extracts. But still, there is a substantial need for an economic, commercially viable and eco-friendly approach, which explores the potential of natural reducing agents to synthesize nanoparticles that is under exploration. Moreover, there is substantial variation in the chemical composition of fruit extracts of the same species of fruits procured from different areas of the world and which could provide us with varying results in different laboratories. Therefore, exploration of biomolecules playing a significant role in nanoparticles synthesis has become an emerging field and an uncovered avenue for research.

Author Contributions: Conceptualization, D.K., F.S., S.K.B., V.K., and K.K.; Manuscript writing, H.K., and K.B.; Manuscript editing, D.S.D., H.R., R.S., and R.V.; Critical revising, D.K., E.N., F.S., S.K.B., V.K., and K.K. All authors have read and agreed to the published version of the manuscript.

Funding: This research was funded by the University of Hradec Kralove (VT2019-2021). Supported also by MH CZ-DRO (UHHK, 00179906).

Acknowledgments: We acknowledge University of Hradec Kralove (VT2019-2021) for financial support. Supported also by MH CZ-DRO (UHHK, 00179906).

Conflicts of Interest: The authors declare no conflict of interest.

\section{References}

1. Bachheti, R.K.; Fikadu, A.; Bachheti, A.; Husen, A. Biogenic fabrication of nanomaterials from flower-based chemical compounds, characterization and their various applications: A review. Saudi J. Biol. Sci. 2020, 27, 2551-2562. [CrossRef] [PubMed]

2. Kalia, A.; Singh, S. Myco-decontamination of azo dyes: Nano-augmentation technologies. 3 Biotech 2020, 10, 384. [CrossRef] [PubMed]

3. Kumar, H.; Bhardwaj, K.; Kuča, K.; Kalia, A.; Nepovimova, E.; Verma, R.; Kumar, D. Flower-based green synthesis of metallic nanoparticles: Applications beyond fragrance. Nanomaterials 2020, 10, 766. [CrossRef] [PubMed]

4. Kumar, H.; Bhardwaj, K.; Sharma, R.; Nepovimova, E.; Kuča, K.; Dhanjal, D.S.; Verma, R.; Bhardwaj, P.; Sharma, S.; Kumar, D. Fruit and vegetable peels: Utilization of high value horticultural waste in novel industrial applications. Molecules 2020, 25, 2812. [CrossRef]

5. Sharma, D.; Kanchi, S.; Bisetty, K. Biogenic synthesis of nanoparticles: A review. Arab J. Chem. 2019, 12, 3576-3600. [CrossRef]

6. Khanna, P.; Kaur, A.; Goyal, D. Algae-based metallic nanoparticles: Synthesis, characterization and applications. J. Microbiol. Methods 2019, 163. [CrossRef]

7. Nadagouda, M.N.; Varma, R.S. A greener synthesis of core (Fe, $\mathrm{Cu}$ )-shell ( $\mathrm{Au}, \mathrm{Pt}, \mathrm{Pd}$, and $\mathrm{Ag}$ ) nanocrystals using aqueous vitamin C. Cryst. Growth Des. 2007, 7, 2582-2587. [CrossRef]

8. Nadagouda, M.N.; Varma, R.S. Microwave-assisted shape-controlled bulk synthesis of noble nanocrystals and their catalytic properties. Cryst. Growth Des. 2007, 7, 686-690.

9. Baruwati, B.; Polshettiwara, V.; Varma, R.S. Glutathione promoted expeditious green synthesis of silver nanoparticles in water using microwaves. Green Chem. 2009, 11, 926-930. [CrossRef] 
10. Polshettiwar, V.; Baruwati, B.; Varma, R.S. Self-assembly of metal oxides into three-dimensional nanostructures: Synthesis and application in catalysis. ACS Nano 2009, 3, 728-736. [CrossRef]

11. Baruwati, B.; Nadagouda, M.N.; Varma, R.S. Bulk synthesis of monodisperse ferrite nanoparticles at water-organic interfaces under conventional and microwave hydrothermal treatment and their surface functionalization. J. Phys. Chem. C 2008, 112, 18399-18404. [CrossRef]

12. Nadagouda, M.N.; Varma, R.S. Green synthesis of silver and palladium nanoparticles at room temperature using coffee and tea extract. Green Chem. 2008, 10, 859-862. [CrossRef]

13. Sidhu, J.S.; Zafar, T.A. Bioactive compounds in banana fruits and their health benefits. Food Qual. Saf. 2018, 2, 183-188. [CrossRef]

14. WHO. Promoting Fruit and Vegetable Consumption Around the World. Available online: https://www.who. int/dietphysicalactivity/fruit/en/ (accessed on 8 June 2020).

15. Chen, L.; Vigneault, C.; Raghavan, G.S.V.; Kubow, S. Importance of the phytochemical content of fruits and vegetables to human health. Stewart Postharvest Rev. 2007, 3, 1-5.

16. Mathews-Roth, M.M. Recent progress in the medical applications of carotenoids. Pure Appl. Chem. 1991, 63, 147-156. [CrossRef]

17. Fraser, P.D.; Bramley, P.M. The biosynthesis and nutritional uses of carotenoids. Prog. Lipid Res. 2004, 43, 228-265. [CrossRef]

18. Clinton, S.K. Lycopene: Chemistry, biology, and implications for human health and disease. Nutr. Rev. 1998, 56, 35-51. [CrossRef]

19. Bramley, P.M. Is lycopene beneficial to human health? Phytochemistry 2000, 54, 233-236. [CrossRef]

20. Giovannucci, E.; Ascherio, A.; Rimm, E.B.; Stampfer, M.J.; Colditz, G.A.; Willett, W.C. Intake of carotenoids and retinol in relation to risk of prostate cancer. J. Natl. Cancer Inst. 1995, 87, 1767-1776. [CrossRef]

21. De Stefani, E.; Oreggia, F.; Boffetta, P.; Deneo-Pellegrini, H.; Ronco, A.; Mendilaharsu, M. Tomatoes, tomato-rich foods, lycopene and cancer of the upper respiratory tract: A case control in Uruguay. Oral Oncol. 2000, 36, 47-53. [CrossRef]

22. Knekt, P.; Jarvinen, R.; Reppanen, R.; Heliovaara, M.; Teppo, L.; Pukkala, E.; Aroma, A. Dietary flavonoids and the risk of lung cancer and other malignant neoplasms. Am. J. Epidemiol. 1997, 146, 223-230. [CrossRef] [PubMed]

23. Gillman, M.W.; Cupples, L.A.; Gagnon, D.; Posner, B.M.; Ellison, R.C.; Castelli, W.P.; Wolf, P.A. Protective effect of fruits and vegetables on development of stroke in men. J. Am. Med. Assoc. 1995, 273, 1113-1117. [CrossRef] [PubMed]

24. Cox, B.D.; Whichelow, M.J.; Prevost, A.T. Seasonal consumption of salad vegetables and fresh fruit in relation to the development of cardiovascular disease and cancer. Public Health Nutr. 2000, 3, 19-29. [CrossRef] [PubMed]

25. Variya, B.C.; Bakrania, A.K.; Patel, S.S. Emblica officinalis (Amla): A review for its phytochemistry, ethnomedicinal uses and medicinal potentials with respect to molecular mechanisms. Pharmacol. Res. 2016, 111, 180-200. [CrossRef]

26. Singh, B.; Singh, J.P.; Kaur, A.; Singh, N. Phenolic compounds as beneficial phytochemicals in pomegranate (Punica granatum L.) peel: A review. Food Chem. 2018, 261, 75-86. [CrossRef]

27. Yugandhar, P.; Vasavi, T.; Rao, Y.J.; Devi, P.U.M.; Narasimha, G.; Savithramma, N. Cost effective, green synthesis of copper oxide nanoparticles using fruit extract of Syzygium alternifolium (Wt.) Walp., characterization and evaluation of antiviral activity. J. Cluster Sci. 2018, 29, 743-755. [CrossRef]

28. Khani, R.; Roostaei, B.; Bagherzade, G.; Moudi, M. Green synthesis of copper nanoparticles by fruit extract of Ziziphus spina-christi (L.) Willd: Application for adsorption of triphenylmethane dye and antibacterial assay. J. Mol. Liq. 2018, 255, 541-549. [CrossRef]

29. Ebrahimi, K.; Shiravand, S.; Mahmoudvand, H. Biosynthesis of copper nanoparticles using aqueous extract of Capparis spinosa fruit and investigation of its antibacterial activity. Marmara Pharm. J. 2017, 21, 866-871. [CrossRef]

30. Hemmati, S.; Ahmeda, A.; Salehabadi, Y.; Zangeneh, A.; Zangeneh, M.M. Synthesis, characterization, and evaluation of cytotoxicity, antioxidant, antifungal, antibacterial, and cutaneous wound healing effects of copper nanoparticles using the aqueous extract of Strawberry fruit and L-Ascorbic acid. Polyhedron. 2020, 180, 114425. [CrossRef] 
31. Carloling, G.; Priyadharshini, M.N.; Vinodhini, E.; Ranjitham, A.M.; Shanthi, P. Biosynthesis of copper nanoparticles using aqueous guava extract-characterisation and study of antibacterial effects. Int. J. Pharm. Biol. Sci. 2015, 5, 25-43.

32. Kaur, P.; Thakur, A.; Chaudhary, A. Biogenesis of copper nanoparticles using peel extract of Punica granatum and their antimicrobial activity against opportunistic pathogens. Green Chem. Lett. Rev. 2016, 9, 33-38. [CrossRef]

33. Gutiérrez, R.M.P.; Mitchell, S.; Solis, R.V. Psidium guajava: A review of its traditional uses, phytochemistry and pharmacology. J. Ethnopharmacol. 2008, 117, 1-27. [CrossRef]

34. Chhikara, N.; Kour, R.; Jaglan, S.; Gupta, P.; Gat, Y.; Panghal, A. Citrus medica: Nutritional, phytochemical composition and health benefits-a review. Food Funct. 2018, 9, 1978-1992. [CrossRef]

35. Dash, S.S.; Bag, B.G. Synthesis of gold nanoparticles using renewable Punica granatum juice and study of its catalytic activity. Appl. Nanosci. 2014, 4, 55-59. [CrossRef]

36. Khan, A.F.; Yuan, Q.; Wei, Y.; Khan, S.U.; Tahir, K.; Khna, Z.U.H.; Ahmad, A.; Ali, F.; Ali, S.; Nazir, S. Longan fruit juice mediated synthesis of uniformly dispersed spherical AuNPs: Cytotoxicity against human breast cancer cell line MCF-7, antioxidant and fluorescent properties. RSC Adv. 2016, 6, 23775-23782. [CrossRef]

37. Gubitosa, J.; Rizzi, V.; Lopedota, A.; Fini, P.; Laurenzana, A.; Fibbi, G.; Fanelli, F.; Petrella, A.; Laquintana, V.; Denora, N.; et al. One pot environmental friendly synthesis of gold nanoparticles using Punica Granatum juice: A novel antioxidant agent for future dermatological and cosmetic applications. J. Colloid Interface Sci. 2018, 521, 50-61. [CrossRef]

38. Yu, J.; Xu, D.; Guan, H.N.; Wang, C.; Huang, L.K.; Chi, D.F. Facile one-step green synthesis of gold nanoparticles using Citrus maxima aqueous extracts and its catalytic activity. Mater. Lett. 2016, 166, 110-112. [CrossRef]

39. Patra, J.K.; Baek, K.H. Novel green synthesis of gold nanoparticles using Citrullus lanatus rind and investigation of proteasome inhibitory activity, antibacterial, and antioxidant potential. Int. J. Nanomed. 2015, 10, 7253-7264.

40. Dauthal, P.; Mukhopadhyay, M. Prunus domestica fruit extract-mediated synthesis of gold nanoparticles and its catalytic activity for 4-nitrophenol reduction. Ind. Eng. Chem. Res. 2012, 51, 13014-13020. [CrossRef]

41. Jassim, A.M.N.; Mohammed, M.T.; Farhan, S.A.; Dadoosh, R.M.; Majeed, Z.N.; Abdula, A.M. Green synthesis of silver nanoparticles using Carica papaya juice and study of their biochemical application. J. Pharm. Sci. Res. 2019, 11, 1025-1034.

42. Edison, T.J.I.; Sethuraman, M.G. Instant green synthesis of silver nanoparticles using Terminalia chebula fruit extract and evaluation of their catalytic activity on reduction of methylene blue. Proc. Biochem. 2012, 47, 1351-1357. [CrossRef]

43. Ramesh, P.S.; Kokila, T.; Geetha, D. Plant mediated green synthesis and antibacterial activity of silver nanoparticles using Emblica Officinalis fruit extract. Spectrochim. Acta A Mol. Biomol. Spectrosc. 2015, 142, 339-343. [CrossRef] [PubMed]

44. Masum, M.M.I.; Siddiqa, M.M.; Ali, K.A.; Zhang, Y.; Abdallah, Y.; Ibrahim, E.; Qiu, W.; Yan, C.; Li, B. Biogenic synthesis of silver nanoparticles using Phyllanthus emblica fruit extract and its inhibitory action against the pathogen Acidovorax oryzae strain RS-2 of rice bacterial brown stripe. Front. Microbiol. 2019, 10, 820. [CrossRef] [PubMed]

45. Jacob, S.J.P.; Prasad, V.L.S.; Sivasankar, S.; Muralidharan, P. Biosynthesis of silver nanoparticles using dried fruit extract of Ficus carica screening for its anticancer activity and toxicity in animal models. Food Chem. Toxicol. 2017, 109, 951-956. [CrossRef]

46. Renuka, R.; Devi, K.R.; Sivakami, M.; Thilagavathi, T.; Uthrakumar, R. Biosynthesis of silver nanoparticles using Phyllanthus emblica fruit extract for antimicrobial application. Biocatal. Agric. Biotechnol. 2020, 24, 101567. [CrossRef]

47. Ebrahimzadeh, M.A.; Naghizadeh, A.; Amiri, O.; Shirzadi-Ahodashti, M. Mortazavi-Derazkola, S. Green and facile synthesis of Ag nanoparticles using Crataegus pentagyna fruit extract (CP-AgNPs) for organic pollution dyes degradation and antibacterial application. Bioorg. Chem. 2020, 94, 103425. [CrossRef]

48. Klimek-Szczykutowicz, M.; Szopa, A.; Ekiert, H. Citrus limon (Lemon) phenomenon-A review of the chemistry, pharmacological properties, applications in the modern pharmaceutical, food, and cosmetics industries, and biotechnological studies. Plants 2020, 9, 119. [CrossRef]

49. Soto, M.L.; Falqué, E.; Domínguez, H. Relevance of natural phenolics from grape and derivative products in the formulation of cosmetics. Cosmetics 2015, 2, 259-276. [CrossRef] 
50. Manzoor, Z.; Nawaz, A.; Mukhtar, H.; Haq, I. Bromelain: Methods of extraction, purification and therapeutic applications. Braz. Arch. Biol. Technol. 2016, 59, e16150010. [CrossRef]

51. Parate, A.M.; Bajpai, N.D.; Walke, D.D. Role of Syzygium cumini (Jamun) in cosmetic. Int. J. Sci. Dev. Res. 2019, 4, 193-201.

52. Aminuzzaman, M.; Ying, L.P.; Goh, W.S.; Watanabe, A. Green synthesis of zinc oxide nanoparticles using aqueous extract of Garcinia mangostana fruit pericarp and their photocatalytic activity. Bull. Mater. Sci. 2018, 41, 50. [CrossRef]

53. Timoszyk, A. A review of the biological synthesis of gold nanoparticles using fruit extracts: Scientific potential and application. Bull. Mater. Sci. 2018, 41, 154. [CrossRef]

54. Shende, S.; Ingle, A.P.; Gade, A.; Rai, M. Green synthesis of copper nanoparticles by Citrus medica Linn. (Idilimbu) juice and its antimicrobial activity. World J. Microbiol. Biotechnol. 2015, 31, 865-873. [CrossRef]

55. Majumdar, M.; Biswas, S.C.; Choudhury, R.; Upadhyay, P.; Adhikary, A.; Roy, D.N.; Misra, T.K. Synthesis of gold nanoparticles using Citrus macroptera fruit extract: Anti-biofilm and anticancer activity. ChemistrySelect 2019, 4, 5714-5723. [CrossRef]

56. Ibrahim, M.H.; Ibrahiem, A.A.; Dalloul, T.R. Biosynthesis of silver nanoparticles using pomegranate juice extract and its antibacterial activity. Int. J. Appl. Sci. Biotechnol. 2016, 4, 254-258. [CrossRef]

57. Gnanajobitha, G.; Paulkumar, K.; Vanaja, M.; Rajeshkumar, S.; Malarkodi, C.; Annadurai, G.; Kannan, C. Fruit-mediated synthesis of silver nanoparticles using Vitis vinifera and evaluation of their antimicrobial efficacy. J. Nanostruct. Chem. 2013, 3, 67. [CrossRef]

58. Zafar, S.; Zafar, A. Biosynthesis and characterization of silver nanoparticles using Phoenix dactylifera fruits extract and their in vitro antimicrobial and cytotoxic effects. Open Biotechnol. J. 2019, 13, 37-46. [CrossRef]

59. Farhadi, S.; Ajerloo, B.; Mohammadi, A. Green biosynthesis of spherical silver nanoparticles by using date palm (Phoenix dactylifera) fruit extract and study of their antibacterial and catalytic activities. Acta Chim. Slov. 2017, 64, 129-143. [CrossRef]

60. Ali, Z.A.; Yahya, R.; Sekaran, S.D.; Puteh, R. Green synthesis of silver nanoparticles using apple extract and its antibacterial properties. Adv. Mater. Sci. Eng. 2016, 2016, 4102196. [CrossRef]

61. Shanmugavadivu, M.; Kuppusamy, S.; Ranjithkumar, R. Synthesis of pomegranate peel extract mediated silver nanoparticles and its antibacterial activity. Am. J. Adv. Drug Deliv. 2014, 2, 174-182.

62. Ibrahim, H.M.M. Green synthesis and characterization of silver nanoparticles using banana peel extract and their antimicrobial activity against representative microorganisms. J. Radiat. Res. Appl. Sci. 2015, 8, 265-275. [CrossRef]

63. Kokila, T.; Ramesh, P.S.; Geetha, D. Biosynthesis of silver nanoparticles from Cavendish banana peel extract and its antibacterial and free radical scavenging assay: A novel biological approach. Appl. Nanosci. 2015, 5, 911-920. [CrossRef]

64. Kahrilas, G.A.; Wally, L.M.; Fredrick, S.J.; Hiskey, M.; Prieto, A.L.; Owens, J.E. Microwave-assisted green synthesis of silver nanoparticles using orange peel extract. ACS Sustain. Chem. Eng. 2014, 2, 367-376. [CrossRef]

65. Ajmal, N.; Saraswat, K.; Sharma, V.; Zafar, M.E. Synthesis and antibacterial activity of silver nanoparticles from Prunus armeniaca (Apricot) fruit peel extract. Bull. Environ. Pharmacol. Life Sci. 2016, 5, 91-94.

66. Devanesan, S.; AlSalh, M.S.; Balaji, R.V.; Ranjitsingh, A.J.A.; Ahamed, A.; Alfuraydi, A.A.; AlQahtani, F.Y.; Aleanizy, F.S.; Othman, A.H. Antimicrobial and cytotoxicity effects of synthesized silver nanoparticles from Punica granatum peel extract. Nanoscale Res. Lett. 2018, 13, 315. [CrossRef]

67. Das, G.; Patra, J.Y.; Debnath, D.; Ansari, A.; Shin, H.S. Investigation of antioxidant, antibacterial, antidiabetic, and cytotoxicity potential of silver nanoparticles synthesized using the outer peel extract of Ananas comosus (L.). PLoS ONE 2019, 14. [CrossRef]

68. He, Y.; Du, Z.; Ma, S.; Cheng, S.; Jiang, S.; Liu, Y.; Li, D.; Huang, H.; Zhang, K.; Zheng, X. Biosynthesis, antibacterial activity and anticancer effects against prostate cancer (pc-3) cells of silver nanoparticles using Dimocarpus longan Lour. peel extract. Nanoscale Res. Lett. 2016, 11, 300. [CrossRef]

69. Pavithra, N.S.; Lingaraju, K.; Raghu, G.K.; Nagaraju, G. Citrus maxima (Pomelo) juice mediated eco-friendly synthesis of $\mathrm{ZnO}$ nanoparticles: Applications to photocatalytic, electrochemical sensor and antibacterial activities. Spectrochim. Acta A Mol. Biomol. Spectrosc. 2017, 185, 11-19. [CrossRef] 
70. Sukri, S.N.A.M.; Shameli, K.; Wong, M.M.T.; Teow, S.Y.; Chew, J.; Ismail, N.F. Cytotoxicity and antibacterial activities of plant-mediated synthesized zinc oxide $(\mathrm{ZnO})$ nanoparticles using Punica granatum (pomegranate) fruit peels extract. J. Mol. Struct. 2019, 1189, 57-65. [CrossRef]

71. Ahmad, R.A.R.; Harun, Z.; Othman, M.H.D.; Basri, H.; Yunos, M.Z.; Ahmad, A.; Akhair, S.H.M.; Rashid, A.Q.A.; Azhar, F.H.; Alias, S.S.; et al. Biosynthesis of zinc oxide nanoparticles by using fruits extracts of Ananas comosus and its antibacterial activity. Malays. J. Fund. Appl. Sci. 2019, 15, 268-273. [CrossRef]

72. Verma, N.; Kumar, N. Synthesis and biomedical applications of copper oxide nanoparticles: An expanding horizon. ACS Biomater. Sci. Eng. 2019, 5, 1170-1188. [CrossRef]

73. Kumar, H.; Bhardwaj, K.; Nepovimova, E.; Kučca, K.; Dhanjal, D.S.; Bhardwaj, S.; Bhatia, S.K.; Verma, R.; Kumar, D. Antioxidant functionalized nanoparticles: A combat against oxidative stress. Nanomaterials 2020, 10, 1334. [CrossRef] [PubMed]

74. Nirmala, J.G.; Akila, S.; Nadar, M.S.A.M.; Narendhirakannan, R.T.; Chatterjee, S. Biosynthesized Vitis vinifera seed gold nanoparticles induce apoptotic cell death in A431 skin cancer cells. RSC Adv. 2016, 6, 82205-82218. [CrossRef]

75. Nirmala, J.G.; Akila, S.; Nadar, M.S.A.M.; Narendhirakannan, R.T.; Chatterjee, S. Vitis vinifera peel polyphenols stabilized gold nanoparticles induce cytotoxicity and apoptotic cell death in A431 skin cancer cell lines. Adv. Powder Technol. 2017, 28, 1170-1184. [CrossRef]

76. Agarwal, H.; Kumar, S.V.; Rajeshkumar, S. A review on green synthesis of zinc oxide nanoparticles -An eco-friendly approach. Res. Effic. Technol. 2017, 3, 406-413. [CrossRef]

77. Jayaseelan, C.; Rahuman, A.A.; Kirthi, A.V.; Marimuthu, S.; Santhoshkumar, T.; Bagavan, A.; Guarav, K.; Karthik, L.; Rao, K.V. Novel microbial route to synthesize ZnO nanoparticles using Aeromonas hydrophila and their activity against pathogenic bacteria and fungi. Spectrochim. Acta A Mol. Biomol. Spectrosc. 2012, 90, 78-84. [CrossRef]

78. Pulit-prociak, J.; Chwastowski, J.; Kucharski, A.; Banach, M. Applied surface science functionalization of textiles with silver and zinc oxide nanoparticles. Appl. Surf. Sci. 2016, 385, 543-553. [CrossRef]

79. Bray, F.; Ferlay, J.; Soerjomataram, I.; Siegel, R.L.; Torre, L.A.; Jemal, A. Global cancer statistics 2018: GLOBOCAN estimates of incidence and mortality worldwide for 36 cancers in 185 countries. CA Cancer J. Clin. 2018, 68, 394-424. [CrossRef]

80. Cragg, G.M.; Newman, D.J. Plants as a source of anticancer agents. J. Ethnopharmacol. 2005, 100, 72-79. [CrossRef]

81. Balunas, M.J.; Kinghorn, A.D. Drug discovery from medicinal plants. Life Sci. 2005, 78, 431-441. [CrossRef]

82. Ren, W.; Qiao, Z.; Wang, H.; Zhu, L.; Zhang, L. Flavonoids: Promising anticancer agents. Med. Res. Rev. 2003, 23, 519-534. [CrossRef]

83. Hu, M.L. Dietary polyphenols as antioxidants and anticancer agents: More questions than answers. Chang. Gung Med. J. 2011, 34, 449-460. [PubMed]

84. Dzubak, P.; Hajduch, M.; Vydra, D.; Hustova, A.; Kvasnica, M.; Biedermann, D.; Markova, L.; Urban, M.; Sarek, J. Pharmacological activities of natural triterpenoids and their therapeutic implications. Nat. Prod. Rep. 2006, 23, 394-411. [CrossRef] [PubMed]

85. Wang, M.; Thanou, M. Targeting nanoparticles to cancer. Pharmacol. Res. 2010, 62, 90-99. [CrossRef]

86. Ratan, Z.A.; Haidere, M.F.; Nurunnabi, M.; Shahriar, S.M.; Ahammad, A.J.S.; Shim, Y.Y.; Reaney, M.J.T.; Cho, J.Y. Green chemistry synthesis of silver nanoparticles and their potential anticancer effects. Cancer 2020, 12, 855. [CrossRef] [PubMed]

87. Wang, L.; Hu, C.; Shao, L. The antimicrobial activity of nanoparticles: Present situation and prospects for the future. Int. J. Nanomed. 2017, 12, 1227-1249. [CrossRef] [PubMed]

88. Lesniak, A.; Salvati, A.; Santos-Martinez, M.J.; Radomski, M.W.; Dawson, K.A.; Åberg, C. Nanoparticle adhesion to the cell membrane and its effect on nano particle uptake efficiency. J. Am. Chem. Soc. 2013, 135, 1438-1444. [CrossRef] [PubMed]

89. Singh, J.; Dutta, T.; Kim, K.H.; Rawat, M.; Samddar, P.; Kumar, P. 'Green' synthesis of metals and their oxidenanoparticles: Applications for environmental remediation. J. Nanobiotechnol. 2018, 16, 84. [CrossRef]

90. Panigrahi, S.; Basu, S.; Praharaj, S.; Pande, S.; Jana, S.; Pal, A.; Ghosh, S.K.; Pal, T. Synthesis and size-selective catalysis by supported gold nanoparticles: Study on heterogeneous and homogeneous catalytic process. J. Phys. Chem. C 2007, 111, 4596-4605. [CrossRef] 
91. Woo, Y.; Lai, D.Y. Aromatic amino and nitro-amino compounds and their halogenated derivatives. In Patty's Toxicology; Bingham, E., Cohrssen, B., Powell, C.H., Eds.; Wiley: Hoboken, NJ, USA, 2012.

92. Sharma, J.K.; Akhtar, M.S.; Ameen, S.; Srivastva, P.; Singh, G. Green synthesis of CuO nanoparticles with leaf extract of Calotropis gigantea and its dye-sensitized solar cells applications. J. Alloys Compd. 2015, 632, 321-325. [CrossRef]

93. Lim, S.H.; Ahn, E.Y.; Park, Y. Green synthesis and catalytic activity of gold nanoparticles synthesized by Artemisia capillaries water extract. Nanoscale Res. Lett. 2016, 11, 474. [CrossRef] [PubMed]

94. Rostami-Vartooni, A.; Nasrollahzadeh, M.; Alizadeh, M. Green synthesis of perlite supported silver nanoparticles using Hamamelis virginiana leaf extract and investigation of its catalytic activity for the reduction of 4-nitrophenol and congo red. J. Alloys Compd. 2016, 680, 309-314. [CrossRef]

95. Gopalakrishnan, R.; Loganathan, B.; Dinesh, S.; Raghu, K. Strategic green synthesis, characterization and catalytic application to 4-nitrophenol reduction of palladium nanoparticles. J. Clust. Sci. 2017, 28, 2123-2131. [CrossRef]

96. Senobari, S.; Nezamzadeh-Ejhieh, A. A comprehensive study on the enhanced photocatalytic activity of CuO-NiO nanoparticles: Designing the experiments. J. Mol. Liq. 2018, 261, 208-217. [CrossRef]

97. Begum, R.; Najeeb, J.; Sattar, A.; Naseem, K.; Irfan, A.; Al-Sehemi, A.G.; Farooqi, Z.H. Chemical reduction of methylene blue in the presence of nanocatalysts: A critical review. Rev. Chem. Eng. 2019, 36. [CrossRef]

Publisher's Note: MDPI stays neutral with regard to jurisdictional claims in published maps and institutional affiliations. 